\title{
Decoupled, Energy Stable Scheme for Hydrodynamic Allen-Cahn Phase Field Moving Contact Line Model ${ }^{*}$
}

\author{
Rui Chen \\ Institute of Applied Physics and Computational Mathematics, Beijing 100088, P.R. China \\ School of Science, Beijing University of Posts and Telecommunications, Beijing 100876, P.R. China \\ Email: ruichenbnu@gmail.com \\ Xiaofeng Yang \\ Department of Mathematics, University of South Carolina, Columbia, SC, 29208, USA \\ Email: xfyang@math.sc.edu \\ Hui Zhang \\ School of Mathematical Sciences, Beijing Normal University, Laboratory of Mathematics and Complex \\ Systems, Ministry of Education, Beijing 100875, P.R. China \\ Email: hzhang@bnu.edu.cn
}

\begin{abstract}
In this paper, we present an efficient energy stable scheme to solve a phase field model incorporating contact line condition. Instead of the usually used Cahn-Hilliard type phase equation, we adopt the Allen-Cahn type phase field model with the static contact line boundary condition that coupled with incompressible Navier-Stokes equations with Navier boundary condition. The projection method is used to deal with the Navier-Stokes equations and an auxiliary function is introduced for the non-convex Ginzburg-Landau bulk potential. We show that the scheme is linear, decoupled and energy stable. Moreover, we prove that fully discrete scheme is also energy stable. An efficient finite element spatial discretization method is implemented to verify the accuracy and efficiency of proposed schemes. Numerical results show that the proposed scheme is very efficient and accurate.
\end{abstract}

Mathematics subject classification: 65N06, 65B99.

Key words: Moving contact line, phase-field, Navier-Stokes equations, Allen-Cahn equation, finite element, energy stable scheme, linear element..

\section{Introduction}

Two phase immiscible flows are common in our lives, such as the air bubble in the water, and droplet of oil in the water, and cells in the blood, etc.. When the air bubble rises to the edge of glass, the interface will change its geometry. Then it will form a moving contact line (MCL) problem. Moving contact line, where the fluid-fluid interface touches the solid wall, has been widely investigated by the researcher theoretically and experimentally. People are interested in the topic that how the contact line evolves when the solid wall moves. In this situation, the no-slip boundary condition for the flow is not applicable again. From this viewpoint, Qian et al. $[28,29]$ proposed the generalized Navier boundary condition (GNBC) according to the molecular dynamics theory. To investigate the complex behavior at MCLs, plenty of models are studied by the researchers. For example, molecular dynamical (MD) simulations are studied by Koplik et al. [15, 16] and Qian et al. [28, 29]. Microscopic-macroscopic hybrid simulations

\footnotetext{
${ }^{*}$ Received xxx / Revised version received xxx / Accepted xxx /
} 
had been carried out by Hadjiconstantinou [8], Ren and E [30] etc. Although this approach is powerful, the computational cost is very expensive for macroscopic applications.

There are a lot of methods to study the MCL problem, such as the immersed interface method [17], the volume of fluid method [31], a hybrid atomistic-continuum method [8], and phase field method [41] etc. In the recent years, phase field method has been used widely on the interfacial phenomena, and has been applied to simulate many dynamical processes successfully in many fields $[2,4,11-14,18-20,22,25,27,35,36,38-43,46-49,51,52,56,61,63,64]$. In a phase field model, a continuous phase field function is used to denote the two immiscible fluids where the fluid-fluid interface has a thickness. In the framework of phase field approach, the governing system is usually obtained from the gradient flow, which is a variational formalism from the total free energy. Thus, there is usually a physical energy law associated with the phase field model [29]. This energy law will help us to carry out mathematical analysis and further design efficient numerical scheme. Thus, from the numerical point view, we are interested in constructing a simple and efficient (linear and decoupled) scheme which satisfies the discrete energy law.

Qian et al. [29] have proposed a Navier-Stokes Cahn-Hilliard (NSCH) system to study the MCL problem, where the GNBC is used for Navier-Stokes equation and the dynamic contact line condition (DCLC) is applied on the Cahn-Hilliard equation. As we all know, the CahnHilliard equation is a four-order equation with volume fraction being conserved, but it needs more time to carry out than Allen-Cahn (second-order) equation on numerical computation. Though Allen-Cahn equation is not conserved on volume fraction, it can keep conserved with the introduction of a Lagrangian multiplier. Thus in this paper we use Allen-Cahn equation to replace the Cahn-Hilliard equation.

The purpose of our paper is to construct a linear, decoupled, fully discrete, first-order, and unconditionally energy stable scheme for the Navier-Stokes Allen-Cahn (NSAC) system. We know that the NSAC system is a coupled system, which includes the coupling between the phase field variable and the velocity in the convection and the stress, and the coupling between the velocity and the pressure in the momentum equation. There are several methods to design the energy stable scheme, such as the operator-splitting on the time-discretization [10], a convex splitting scheme in [1,33], and the stabilization approach in [21,22,24,36-39]. Although these schemes are energy stable, they still have some disadvantages. Firstly, the operatorsplitting scheme and the convex splitting scheme are usually coupled and nonlinear due to the couplings in the system, which take much time on iterations to carry out the numerical results. Secondly, it is difficult to prove the unconditional solvability for these nonlinear schemes. At last, although the stabilizing approach is linear and decoupled, the truncation error is introduced in the scheme, which requires the double well potential to be bounded form the viewpoint of mathematics. Thus, in this paper, we shall overcome these difficulties to construct a fully discrete, linear and decoupled energy stable scheme using the recently developed "Invariant Energy Quadratization" approach [5, 9, 50,53-55, 57,58,60,62]. In our previous paper [24,59], we developed some decoupled, energy stable numerical schemes to solve the hydrodynamics coupled Allen-Cahn MCL phase field model by adding the extra stabilizing terms for phase field equation. In this paper we adopt a novel skill to construct a linear, decoupled, and unconditionally energy stable scheme without using the stabilizing approach for the phase field equation.

The rest of the paper is organized as follows. In the next section, we present the phase field model of moving contact line condition and show the energy dissipation law for the system. In 
section 3 , we construct a linear decoupled energy stable scheme for this coupled nonlinear system by the introduction of the auxiliary function. In section 4, we implement the finite element method on this model for spatial discretization and prove that the fully discrete scheme is energy stable. In section 5, we present some numerical simulations to illustrate the efficiency and accuracy of the proposed numerical scheme. Finally, we give some conclusions in the last section.

\section{A Navier-Stokes Allen-Cahn coupled model}

In $[28,29]$, the Navier-Stokes Cahn-Hilliard coupled system (NSCH) with a GNBC was developed to study the two-phase incompressible, immiscible fluid, where the fluid-fluid interface touches the solid wall. A non-dimensional version of the system is given as follows.

Incompressible Navier-Stokes equations for hydrodynamics

$$
\begin{aligned}
& \mathbf{u}_{t}+(\mathbf{u} \cdot \nabla) \mathbf{u}=\nu \Delta \mathbf{u}-\nabla p+\lambda \mu \nabla \phi, \\
& \nabla \cdot \mathbf{u}=0, \\
& \mathbf{u} \cdot \mathbf{n}=0, \quad \text { on } \quad \partial \Omega, \\
& l(\phi)\left(\mathbf{u}_{\tau}-\mathbf{u}_{w}\right)+\nu \partial_{\mathbf{n}} \mathbf{u}_{\tau}-\lambda L(\phi) \nabla_{\tau} \phi=0, \quad \text { on } \quad \partial \Omega .
\end{aligned}
$$

Cahn-Hilliard type phase field equations:

$$
\begin{aligned}
& \phi_{t}+\mathbf{u} \cdot \nabla \phi=M \Delta \mu, \\
& \mu=-\varepsilon \Delta \phi+f(\phi), \\
& \partial_{\mathbf{n}} \mu=0, \quad \text { on } \partial \Omega, \\
& \phi_{t}+\mathbf{u}_{\tau} \cdot \nabla_{\tau} \phi=-\gamma L(\phi), \quad \text { on } \quad \partial \Omega,
\end{aligned}
$$

where $\mathbf{u}$ is the fluid velocity, $p$ is the pressure, $\phi$ is the phase field variable, $\mu$ is the chemical potential, the function $L(\phi)$ is given by

$$
L(\phi)=\varepsilon \partial_{\mathbf{n}} \phi+g^{\prime}(\phi),
$$

where $g(\phi)$ is the boundary interfacial energy, $l(\phi) \geq 0$ is a given coefficient function. The function $f(\phi)=F^{\prime}(\phi)$ with $F(\phi)$ being the Ginzburg-Landau bulk potential. More precisely, $F(\phi)$ and $g(\phi)$ are defined as

$$
F(\phi)=\frac{1}{4 \varepsilon}\left(\phi^{2}-1\right)^{2}, \quad g(\phi)=-\frac{\sqrt{2}}{3} \cos \theta_{s} \sin \left(\frac{\pi}{2} \phi\right),
$$

where $\theta_{s}$ is the static contact angle. In equations (2.1)-(2.8), $\nabla$ denote the gradient operator, $\mathbf{n}$ is the outward normal direction on boundary $\partial \Omega, \tau$ is tangential direction on the boundary, and vector operator $\nabla_{\tau}=\nabla-(\mathbf{n} \cdot \nabla) \mathbf{n}$ is the gradient along tangential direction, $\mathbf{u}_{w}$ is the boundary wall velocity, $\mathbf{u}_{\tau}$ is the boundary fluid velocity in tangential direction. From (2.3), we have $\mathbf{u}=\mathbf{u}_{\tau}$ on boundary $\partial \Omega$. There are six non-dimensional parameters in this system. $\nu$ is the viscosity coefficient, $\lambda$ denotes the strength of the capillary force comparing to the Newtonian fluid stress, $M$ is the mobility coefficient, $\gamma$ is a boundary relaxation coefficient, $l(\phi)$ is the ratio of domain size to boundary slip length, $\varepsilon$ denotes the interface thickness.

When $\gamma \rightarrow+\infty$, the DCLC (2.8) reduces to static contact line condition (SCLC),

$$
L(\phi)=0, \quad \text { on } \quad \partial \Omega,
$$


and the GNBC reduces to the Navier boundary condition (NBC),

$$
l(\phi)\left(\mathbf{u}_{\tau}-\mathbf{u}_{w}\right)+\nu \partial_{\mathbf{n}} \mathbf{u}_{\tau}=0, \quad \text { on } \quad \partial \Omega .
$$

If we further set $g^{\prime}(\phi) \equiv 0$, the SCLC (2.11) turns to the Neumann boundary condition. If we take $l(\phi) \rightarrow+\infty$ in (2.12), then the Navier slip boundary condition reduces to traditional no-slip boundary condition.

In this paper, we replace the fourth order Cahn-Hilliard equation with the second order Allen-Cahn equation with a Lagrangian multiplier. In this new Navier-Stokes Allen-Cahn (NSAC) system, the fluid equation is same as (2.1)-(2.4). The non-dimensional, Allen-Cahn phase field equation is given as follows.

$$
\begin{aligned}
& \phi_{t}+\mathbf{u} \cdot \nabla \phi=-M \mu, \\
& \mu=-\varepsilon \Delta \phi+f(\phi), \\
& \phi_{t}+\mathbf{u}_{\tau} \cdot \nabla_{\tau} \phi=-\gamma L(\phi), \quad \text { on } \quad \partial \Omega .
\end{aligned}
$$

To preserve the volume fraction, we add the Lagrangian multiplier $\xi(t)$ as follows.

$$
\begin{aligned}
& \mu=-\varepsilon \Delta \phi+f(\phi)+\xi(t), \\
& \frac{d}{d t} \int_{\Omega} \phi d x=0 .
\end{aligned}
$$

Remark 2.1. By the integration of (2.13) on $\Omega$, we can obtain the Lagrangian multiplier $\xi(t)$ depending on $t$,

$$
\xi(t)=-\frac{1}{|\Omega|}\left(\int_{\Omega} f(\phi) d x-\int_{\partial \Omega} \varepsilon \partial_{\mathbf{n}} \phi d s\right)
$$

We now derive the energy dissipation law for PDEs system (2.1)-(2.4) and (2.13), (2.15), (2.16). Here and after, for any function $f, g \in L^{2}(\Omega)$, we use $(f, g)$ to denote $\int_{\Omega} f g d x,(f, g)_{\partial \Omega}$ to denote $\int_{\partial \Omega} f g d x$, and $\|f\|^{2}=(f, f)$ and $\|f\|_{\partial \Omega}^{2}=(f, f)_{\partial \Omega}$.

Theorem 2.1. The NSAC system (2.1)-(2.4) and (2.13), (2.15), (2.16) with GNBC (2.4) and $D C L C(2.8)$ is a dissipative system satisfying the following energy dissipation law,

$$
\frac{d E_{t o t}}{d t}=-\nu\|\nabla \mathbf{u}\|^{2}-\lambda M\|\mu\|^{2}-\lambda \gamma\|L(\phi)\|_{\partial \Omega}^{2}-\left\|l(\phi)^{\frac{1}{2}} \mathbf{u}_{s}\right\|_{\partial \Omega}^{2}-\left(l(\phi) \mathbf{u}_{s}, \mathbf{u}_{w}\right)_{\partial \Omega},
$$

where $\mathbf{u}_{s}=\mathbf{u}_{\tau}-\mathbf{u}_{w}$ is the velocity slip on boundary $\partial \Omega, E_{t o t}=E_{k}[\mathbf{u}]+E_{b}[\phi]+E_{s}[\phi]$, and

$$
E_{k}[\mathbf{u}]=\frac{\|\mathbf{u}\|^{2}}{2}, \quad E_{b}[\phi]=\lambda \varepsilon \frac{\|\nabla \phi\|^{2}}{2}+\lambda(F(\phi), 1), \quad E_{s}[\phi]=\lambda(g(\phi), 1)_{\partial \Omega} .
$$

Proof. By taking the inner product of (2.1) with $\mathbf{u}$, using the incompressible condition (2.2) and the zero flux boundary condition (2.3), we have

$$
\frac{1}{2} \frac{d}{d t}\|\mathbf{u}\|^{2}=\nu\left(\partial_{\mathbf{n}} \mathbf{u}, \mathbf{u}\right)_{\partial \Omega}-\nu\|\nabla \mathbf{u}\|^{2}+\lambda(\mu \nabla \phi, \mathbf{u}) .
$$

By taking the inner product of (2.13) with $\lambda \mu$, we get

$$
\lambda\left(\phi_{t}, \mu\right)+\lambda(\mathbf{u} \cdot \nabla \phi, \mu)=-\lambda M\|\mu\|^{2} .
$$


By taking the inner product of $(2.16)$ with $\lambda \phi_{t}$, we have

$$
\lambda\left(\mu, \phi_{t}\right)=-\lambda \varepsilon\left(\partial_{\mathbf{n}} \phi, \phi_{t}\right)_{\partial \Omega}+\frac{1}{2} \lambda \varepsilon \frac{d}{d t}\|\nabla \phi\|^{2}+\lambda \frac{d}{d t}(F(\phi), 1) .
$$

Combining equations (2.21)-(2.23), we obtain

$$
\begin{aligned}
\frac{1}{2} \frac{d}{d t}\|\mathbf{u}\|^{2}+\frac{1}{2} \lambda \varepsilon \frac{d}{d t}\|\nabla \phi\|^{2}+\lambda \frac{d}{d t}(F(\phi), 1)= & -\nu\|\nabla \mathbf{u}\|^{2}-\lambda M\|\mu\|^{2} \\
& +\nu\left(\partial_{\mathbf{n}} \mathbf{u}, \mathbf{u}\right)_{\partial \Omega}+\lambda \varepsilon\left(\partial_{\mathbf{n}} \phi, \phi_{t}\right)_{\partial \Omega} .
\end{aligned}
$$

Then, by using (2.8), (2.9) and boundary condition (2.4), we have

$$
\begin{aligned}
\nu\left(\partial_{\mathbf{n}} \mathbf{u}, \mathbf{u}\right)_{\partial \Omega}=\nu\left(\partial_{\mathbf{n}} \mathbf{u}_{\tau}, \mathbf{u}_{\tau}\right)_{\partial \Omega} & =\left(\lambda L(\phi) \nabla_{\tau} \phi-l(\phi)\left(\mathbf{u}_{\tau}-\mathbf{u}_{w}\right), \mathbf{u}_{\tau}\right)_{\partial \Omega} \\
& =\lambda\left(L(\phi) \nabla_{\tau} \phi, \mathbf{u}_{\tau}\right)_{\partial \Omega}-\left(l(\phi) \mathbf{u}_{s}, \mathbf{u}_{s}+\mathbf{u}_{w}\right)_{\partial \Omega}
\end{aligned}
$$

and

$$
\begin{aligned}
\lambda \varepsilon\left(\partial_{\mathbf{n}} \phi, \phi_{t}\right)_{\partial \Omega} & =\lambda\left(L(\phi)-g^{\prime}(\phi), \phi_{t}\right)_{\partial \Omega} \\
& =\lambda\left(L(\phi), \phi_{t}\right)_{\partial \Omega}-\lambda\left(g^{\prime}(\phi), \phi_{t}\right)_{\partial \Omega} \\
& =\lambda\left(L(\phi),-\mathbf{u}_{\tau} \cdot \nabla_{\tau} \phi-\gamma L(\phi)\right)_{\partial \Omega}-\lambda \frac{d}{d t}(g(\phi), 1)_{\partial \Omega} \\
& =-\lambda\left(L(\phi) \nabla_{\tau} \phi, \mathbf{u}_{\tau}\right)_{\partial \Omega}-\lambda \gamma\|L(\phi)\|_{\partial \Omega}^{2}-\lambda \frac{d}{d t}(g(\phi), 1)_{\partial \Omega} .
\end{aligned}
$$

Summing up (2.24), (2.25) and (2.26), we obtain the energy desired energy estimate (2.19).

Even though the above PDE energy law is straightforward, the nonlinear terms in $\mu$ involves the second order derivatives, and it is not convenient to use them as test functions in numerical approximations, making it difficult to prove the discrete energy dissipation law. To overcome this difficulty, we have to reformulate the momentum equation (2.1) in an alternative form which is convenient for numerical approximation, we let $\dot{\phi}=\phi_{t}+\mathbf{u} \cdot \nabla \phi$, and notice that $\mu=\frac{1}{-M} \dot{\phi}$, then (2.1) can be rewritten as the following equivalent form,

$$
\mathbf{u}_{t}+(\mathbf{u} \cdot \nabla) \mathbf{u}=\nu \Delta \mathbf{u}-\nabla p-\frac{\lambda}{M} \dot{\phi} \nabla \phi
$$

This equivalent form (2.27)-(2.2)-(2.3)-(2.4) and (2.13), (2.15), (2.16) still admits the similar energy law. Taking the inner product of (2.27) with $\mathbf{u},(2.13)$ with $\frac{\lambda}{M} \phi_{t}$ and (2.16) with $\lambda \phi_{t}$, we derive

$$
\begin{gathered}
\frac{1}{2} \frac{d}{d t}\|\mathbf{u}\|^{2}=\nu\left(\partial_{\mathbf{n}} \mathbf{u}, \mathbf{u}\right)_{\partial \Omega}-\nu\|\nabla \mathbf{u}\|^{2}-\frac{\lambda}{M}(\dot{\phi} \nabla \phi, \mathbf{u}) . \\
\frac{\lambda}{M}\|\dot{\phi}\|^{2}-\frac{\lambda}{M}(\dot{\phi}, \mathbf{u} \cdot \nabla \phi)=-\lambda\left(\mu, \phi_{t}\right) . \\
\lambda\left(\mu, \phi_{t}\right)=-\lambda \varepsilon\left(\partial_{\mathbf{n}} \phi, \phi_{t}\right)_{\partial \Omega}+\frac{1}{2} \lambda \varepsilon \frac{d}{d t}\|\nabla \phi\|^{2}+\lambda \frac{d}{d t}(F(\phi), 1) .
\end{gathered}
$$

Taking the summation of the above equalities, we have

$$
\begin{aligned}
\frac{1}{2} \frac{d}{d t}\|\mathbf{u}\|^{2}+\frac{1}{2} \lambda \varepsilon \frac{d}{d t}\|\nabla \phi\|^{2}+\lambda \frac{d}{d t}(F(\phi), 1)= & -\nu\|\nabla \mathbf{u}\|^{2}-\frac{\lambda}{M}\|\dot{\phi}\|^{2} \\
& +\nu\left(\partial_{\mathbf{n}} \mathbf{u}, \mathbf{u}\right)_{\partial \Omega}+\lambda \varepsilon\left(\partial_{\mathbf{n}} \phi, \phi_{t}\right)_{\partial \Omega} .
\end{aligned}
$$


Using (2.25) and (2.26), we have the energy dissipation law.

$$
\frac{d E_{t o t}}{d t}=-\nu\|\nabla \mathbf{u}\|^{2}-\frac{\lambda}{M}\|\dot{\phi}\|^{2}-\lambda \gamma\|L(\phi)\|_{\partial \Omega}^{2}-\left\|l(\phi)^{\frac{1}{2}} \mathbf{u}_{s}\right\|_{\partial \Omega}^{2}-\left(l(\phi) \mathbf{u}_{s}, \mathbf{u}_{w}\right)_{\partial \Omega} .
$$

We emphasize that the above derivation is suitable in a finite dimensional approximation since the test function $\phi_{t}$ is in the same subspaces as $\phi$. Hence, it allows us to design numerical schemes which satisfy the energy dissipation law in the discrete level.

In Shen et al. paper [41] which focus on the NSCH system, for the SCLC (2.11) and NBC (2.12), a linear decoupled energy stable scheme is developed. Meanwhile, for the DCLC (2.8) and GNBC (2.4), in order to obtain the energy stability, only a linear coupled scheme can be developed. To the best of the author's knowledge, there exist some essential difficulties to obtain the estimates for a boundary term, thus it seems formidable to obtain the decoupled energy stable scheme for DCLC coupled with GNBC.

Similar to [41], in this paper, we only develop the numerical scheme for the NSAC system with SCLC (2.11) and NBC (2.12). For the convenience of reading, we list the whole model as follows.

The hydrodynamics equation with NBC

$$
\begin{aligned}
& \mathbf{u}_{t}+(\mathbf{u} \cdot \nabla) \mathbf{u}=\nu \Delta \mathbf{u}-\nabla p-\frac{\lambda}{M} \dot{\phi} \nabla \phi \\
& \nabla \cdot \mathbf{u}=0, \\
& \mathbf{u} \cdot \mathbf{n}=0, \quad \text { on } \quad \partial \Omega . \\
& l(\phi)\left(\mathbf{u}_{\tau}-\mathbf{u}_{w}\right)+\nu \frac{\partial \mathbf{u}_{\tau}}{\partial \mathbf{n}}=0, \quad \text { on } \quad \partial \Omega .
\end{aligned}
$$

The Allen-Cahn type phase field equation with SCLC

$$
\begin{aligned}
& \phi_{t}+(\mathbf{u} \cdot \nabla) \phi=M(\varepsilon \Delta \phi-f(\phi)-\xi(t)), \\
& \frac{d}{d t} \int_{\Omega} \phi d x=0, \\
& L(\phi)=0, \quad \text { on } \quad \partial \Omega .
\end{aligned}
$$

Remark 2.2. There exists some essential difficulties to obtain the estimates for a boundary term for the GNBC and DCLC. In order to obtain the decoupled energy stable scheme for the system, we only consider the $N B C$ and $S C L C$ here.

\section{A Linear, Decoupled Energy Stable Numerical Scheme}

We aim to construct a linear, decoupled energy stable scheme. We know that $g(\phi)$ is bounded and give the following definition

$$
\bar{L}:=\max _{\phi \in \mathbb{R}}\left|g^{\prime \prime}(\phi)\right|=\frac{\sqrt{2} \pi^{2}}{12}\left|\cos \theta_{s}\right| .
$$

In this section we want to construct an energy stable linear scheme which is first-order temporal accurate. One introduces a function $q=\left(\phi^{2}-1\right) / \varepsilon$ such that one can write $f(\phi)=q \phi$. Then it follows that $\partial_{t} q=2 \phi \partial_{t} \phi / \varepsilon$. By using the function $q$, the total energy can be written as

$$
E_{t o t}=\frac{1}{2}\|\mathbf{u}\|^{2}+\lambda \varepsilon \frac{\|\nabla \phi\|^{2}}{2}+\lambda \varepsilon \frac{\|q\|^{2}}{4}+\lambda(g(\phi), 1)_{\partial \Omega} .
$$


The energy laws (2.19) and (2.32) still hold.

Let $\delta t>0$ be a time discretization step and suppose $\mathbf{u}^{n}, \phi^{n}$ and $p^{n}$ are given, where superscript $n$ on variables denotes approximations of corresponding variables at time $n \delta t$.

A first-order linear decoupled (LD) scheme for solving the PDE system (2.33)-(2.39) is constructed as follows.

Given the initial conditions $\phi^{0}, \mathbf{u}^{0}, q^{0}=\left(\left(\phi^{0}\right)^{2}-1\right) / \varepsilon$ and $p^{0}=0$, we aim to compute $\phi^{n+1}$, $q^{n+1}, \tilde{\mathbf{u}}^{n+1}, \mathbf{u}^{n+1}, p^{n+1}$ by having computed $\phi^{n}, q^{n}, \mathbf{u}^{n}, p^{n}$ for $n \geq 0$.

Step 1:

$$
\begin{aligned}
& \dot{\phi}^{n+1}=M\left(\varepsilon \Delta \phi^{n+1}-\phi^{n} q^{n+1}-\xi^{n}\right), \\
& \int_{\Omega}\left(\phi^{n+1}-\phi^{n}\right) d x=0, \\
& \frac{\varepsilon}{2} \frac{q^{n+1}-q^{n}}{\delta t}=\phi^{n} \frac{\phi^{n+1}-\phi^{n}}{\delta t},
\end{aligned}
$$

with boundary condition

$$
\tilde{L}^{n+1}=\varepsilon \partial_{\mathbf{n}} \phi^{n+1}+g^{\prime}\left(\phi^{n}\right)+S\left(\phi^{n+1}-\phi^{n}\right)=0,
$$

where

$$
\mathbf{u}_{\star}^{n}=\mathbf{u}^{n}-\frac{\lambda}{M} \delta t \dot{\phi}^{n+1} \nabla \phi^{n}, \quad \dot{\phi}^{n+1}=\frac{\phi^{n+1}-\phi^{n}}{\delta t}+\left(\mathbf{u}_{\star}^{n} \cdot \nabla\right) \phi^{n} .
$$

Step 2:

$$
\frac{\tilde{\mathbf{u}}^{n+1}-\mathbf{u}_{\star}^{n}}{\delta t}-\nu \Delta \tilde{\mathbf{u}}^{n+1}+B\left(\mathbf{u}^{n}, \tilde{\mathbf{u}}^{n+1}\right)+\nabla p^{n}=0,
$$

with boundary condition

$$
\tilde{\mathbf{u}}^{n+1} \cdot \mathbf{n}=0, \quad \nu \partial_{\mathbf{n}} \tilde{\mathbf{u}}_{\tau}^{n+1}+l\left(\phi^{n}\right) \tilde{\mathbf{u}}_{s}^{n+1}=0,
$$

where

$$
B(\mathbf{u}, \mathbf{v})=(\mathbf{u} \cdot \nabla) \mathbf{v}+\frac{1}{2}(\nabla \cdot \mathbf{u}) \mathbf{v} .
$$

Step 3:

$$
\begin{aligned}
& \frac{\mathbf{u}^{n+1}-\tilde{\mathbf{u}}^{n+1}}{\delta t}+\nabla\left(p^{n+1}-p^{n}\right)=0, \\
& \nabla \cdot \mathbf{u}^{n+1}=0, \\
& \mathbf{u}^{n+1} \cdot \mathbf{n}=0, \quad \text { on } \quad \partial \Omega .
\end{aligned}
$$

In the above, $S$ is a parameter to be determined.

Several remarks are in order.

Remark 3.1. A pressure-correction scheme is used to decouple the computation of the pressure from that of the velocity. About the overview of the projection type methods, we refer to [6, 7, 34].

Remark 3.2. $B(\mathbf{u}, \mathbf{v})$ is the skew-symmetric form of the nonlinear advection term is the Navier-Stokes equation, which is first introduced by Témam [45]. If the velocity is divergence 
free, then $B(\mathbf{u}, \mathbf{u})=(\mathbf{u} \cdot \nabla) \mathbf{u}$. In our scheme $\tilde{\mathbf{u}}^{n+1}$ is not divergence free, but we notice that the following identity

$$
(B(\mathbf{u}, \mathbf{v}), \mathbf{v})=0, \quad \text { if }\left.\quad \mathbf{u} \cdot \mathbf{n}\right|_{\partial \Omega}=0
$$

Thus this identity holds regardless of whether $\mathbf{u}$ or $\mathbf{v}$ are divergence free or not, which would help to preserve the discrete energy stability.

Remark 3.3. Inspired by [3, 4, 26,38,39,41], we introduce the explicit convective velocity $\mathbf{u}_{\star}^{n}$ in (3.3) by combining the term $\mathbf{u}^{n}$ with surface tension term $\dot{\phi} \nabla \phi$. From (3.7), we obtain

$$
\mathbf{u}_{\star}^{n}=\tilde{B}^{-1}\left(\mathbf{u}^{n}-\frac{\phi^{n+1}-\phi^{n}}{M / \lambda} \nabla \phi^{n}\right),
$$

where $\tilde{B}=\left(I+\frac{\delta t}{M / \lambda} \nabla \phi^{n} \nabla \phi^{n}\right)$. It is easy to get the $\operatorname{det}(I+c \nabla \phi \nabla \phi)=1+c \nabla \phi \cdot \nabla \phi$, thus $\tilde{B}$ is invertible.

Remark 3.4. The scheme (3.3)-(3.13) is totally decoupled, linear, and first-order in time. (3.11) can be reformulated as a Poisson equation for $p^{n+1}-p^{n}$. Therefor, at each time step, one only needs to solve a sequence of a decoupled elliptic equations which can be solved very efficiently.

Next we shall show below, the above scheme is unconditionally energy stable.

Theorem 3.1. Assuming $\mathbf{u}_{w}=\mathbf{0}$ and $S \geq \bar{L} / 2$, the solution of (3.3)-(3.13) satisfies the following discrete energy law

$$
\begin{aligned}
& E_{\text {tot }}^{n+1}+\frac{\delta t^{2}}{2}\left\|\nabla p^{n+1}\right\|^{2}+\delta t\left[\nu\left\|\nabla \tilde{\mathbf{u}}^{n+1}\right\|^{2}+\frac{\lambda}{M}\left\|\dot{\phi}^{n+1}\right\|^{2}+\left\|l^{1 / 2}\left(\phi^{n}\right) \tilde{\mathbf{u}}_{s}^{n+1}\right\|_{\partial \Omega}^{2}\right] \\
& \leq E_{t o t}^{n}+\frac{\delta t^{2}}{2}\left\|\nabla p^{n}\right\|^{2}, \quad n=0,1,2, \cdots,
\end{aligned}
$$

where $E_{\text {tot }}^{n}=\left\|\mathbf{u}^{n}\right\|^{2} / 2+\lambda\left(\varepsilon\left\|\nabla \phi^{n}\right\|^{2} / 2+\varepsilon\left\|q^{n}\right\|^{2} / 4+\left(g\left(\phi^{n}, 1\right)\right)_{\partial \Omega}\right)$.

Proof. By taking the $L^{2}$ inner product of (3.3) with $\frac{\lambda}{M} \frac{\phi^{n+1}-\phi^{n}}{\delta t}$, and performing integration by parts, we obtain

$$
\begin{aligned}
& \frac{\lambda}{M}\left\|\dot{\phi}^{n+1}\right\|^{2}-\frac{\lambda}{M}\left(\dot{\phi}^{n+1}, \mathbf{u}_{\star}^{n} \cdot \nabla \phi^{n}\right)-\frac{\lambda}{\delta t}\left(\varepsilon \partial_{\mathbf{n}} \phi^{n+1}, \phi^{n+1}-\phi^{n}\right)_{\partial \Omega} \\
& +\frac{\lambda \varepsilon}{\delta t}\left(\frac{1}{2}\left\|\nabla \phi^{n+1}\right\|^{2}-\frac{1}{2}\left\|\nabla \phi^{n}\right\|^{2}+\left\|\nabla\left(\phi^{n+1}-\phi^{n}\right)\right\|^{2}\right)+\frac{\lambda}{\delta t}\left(\phi^{n} q^{n+1}, \phi^{n+1}-\phi^{n}\right)=0,
\end{aligned}
$$

where we have used the identity

$$
(a-b, 2 a)=|a|^{2}-|b|^{2}+|a-b|^{2} .
$$

The boundary term

$$
\begin{aligned}
-\frac{\lambda}{\delta t}\left(\varepsilon \partial_{\mathbf{n}} \phi^{n+1}, \phi^{n+1}-\phi^{n}\right)_{\partial \Omega} & =\frac{\lambda}{\delta t}\left[\left(g\left(\phi^{n+1}\right), 1\right)_{\partial \Omega}+\left(g\left(\phi^{n}\right), 1\right)_{\partial \Omega}\right. \\
& \left.-\left(S-\frac{g^{\prime \prime}(\zeta)}{2},\left(\phi_{n+1}-\phi_{n}\right)^{2}\right)_{\partial \Omega}\right],
\end{aligned}
$$


where we have used the Taylor-expansion

$$
g^{\prime}\left(\phi^{n}\right)\left(\phi^{n+1}-\phi^{n}\right)=g\left(\phi^{n+1}\right)-g\left(\phi^{n}\right)-\frac{g^{\prime \prime}(\zeta)}{2}\left(\phi^{n+1}-\phi^{n}\right)^{2} .
$$

Taking the $L^{2}$ inner product of (3.5) with $\lambda q^{n+1}$, one obtains

$$
\frac{\lambda \varepsilon}{4 \delta t}\left(\left\|q^{n+1}\right\|^{2}-\left\|q^{n}\right\|^{2}+\left\|q^{n+1}-q^{n}\right\|^{2}\right)-\frac{\lambda}{\delta t}\left(\phi^{n}\left(\phi^{n+1}-\phi^{n}\right), q^{n+1}\right)=0 .
$$

By taking the inner product of equation (3.7) with $\mathbf{u}_{\star}^{n} / \delta t$, we obtain

$$
\frac{1}{2 \delta t}\left(\left\|\mathbf{u}_{\star}^{n}\right\|^{2}-\left\|\mathbf{u}^{n}\right\|^{2}+\left\|\mathbf{u}_{\star}^{n}-\mathbf{u}^{n}\right\|^{2}\right)=-\frac{\lambda}{M}\left(\dot{\phi}^{n+1} \cdot \nabla \phi^{n}, \mathbf{u}_{\star}^{n}\right) .
$$

Taking the $L^{2}$ inner product of (3.8) with $\tilde{\mathbf{u}}^{n+1}$, performing integration by parts, and using identity (3.14), we obtain

$$
\begin{aligned}
\frac{1}{2 \delta t}\left(\left\|\tilde{\mathbf{u}}^{n+1}\right\|^{2}-\left\|\mathbf{u}_{\star}^{n}\right\|^{2}\right. & \left.+\left\|\tilde{\mathbf{u}}^{n+1}-\mathbf{u}_{\star}^{n}\right\|^{2}\right)+\nu\|\nabla \tilde{\mathbf{u}}\|^{2}+\left(\nabla p^{n}, \tilde{\mathbf{u}}^{n+1}\right) \\
& -\nu\left(\partial_{\mathbf{n}} \tilde{\mathbf{u}}^{n+1}, \tilde{\mathbf{u}}^{n+1}\right)_{\partial \Omega}=0 .
\end{aligned}
$$

For the boundary term in the above equation, noticing that $\tilde{\mathbf{u}}_{\tau}^{n+1}-\tilde{\mathbf{u}}_{w}^{n+1}=\tilde{\mathbf{u}}_{s}^{n+1}, \tilde{\mathbf{u}}_{w}^{n+1}=0$, we have

$$
\nu\left(\partial_{\mathbf{n}} \tilde{\mathbf{u}}^{n+1}, \tilde{\mathbf{u}}^{n+1}\right)_{\partial \Omega}=-\left\|l^{1 / 2}\left(\phi^{n}\right) \tilde{\mathbf{u}}_{s}^{n+1}\right\|_{\partial \Omega}^{2} .
$$

By taking the $L^{2}$ inner product of (3.11) with $\mathbf{u}^{n+1}$ and performing integration by parts, and using the incompressible condition (3.12), we have

$$
\frac{1}{2 \delta t}\left(\left\|\mathbf{u}^{n+1}\right\|^{2}-\left\|\tilde{\mathbf{u}}^{n+1}\right\|^{2}+\left\|\mathbf{u}^{n+1}-\tilde{\mathbf{u}}^{n+1} \mid\right\|^{2}\right)=0
$$

By taking the inner product of (3.11) with $\delta t \nabla p^{n}$, and using the condition (3.12), we obtain

$$
\frac{\delta t}{2}\left(\left\|\nabla p^{n+1}\right\|^{2}-\left\|\nabla p^{n}\right\|^{2}-\left\|\nabla\left(p^{n+1}-p^{n}\right)\right\|^{2}\right)-\left(\tilde{\mathbf{u}}^{n+1}, \nabla p^{n}\right)=0 .
$$

We can also obtain the following equation directly from the equation (3.11),

$$
\frac{\delta t}{2}\left\|\nabla p^{n+1}-\nabla p^{n}\right\|^{2}=\frac{1}{2 \delta t}\left\|\tilde{\mathbf{u}}^{n+1}-\mathbf{u}^{n+1}\right\|^{2} .
$$

Hence, combing (3.17), (3.19), (3.21), (3.22), (3.23), (3.24), (3.25), (3.26) and (3.27), we get

$$
\begin{aligned}
\frac{1}{\delta t}\left(E_{\text {tot }}^{n+1}-E_{\text {tot }}^{n}\right) & +\frac{\delta t}{2}\left(\left\|\nabla p^{n+1}\right\|^{2}-\left\|\nabla p^{n}\right\|^{2}\right)= \\
& -\left[\nu\left\|\nabla \tilde{\mathbf{u}}^{n+1}\right\|^{2}+\frac{\lambda}{M}\left\|\dot{\phi}^{n+1}\right\|^{2}+\left\|l^{1 / 2}\left(\phi^{n}\right) \tilde{\mathbf{u}}_{s}^{n+1}\right\|_{\partial \Omega}^{2}\right] \\
& -\frac{1}{2 \delta t}\left(\left\|\tilde{\mathbf{u}}^{n+1}-\mathbf{u}_{\star}^{n}\right\|^{2}+\left\|\mathbf{u}_{\star}^{n}-\mathbf{u}^{n}\right\|^{2}\right)-\frac{\lambda \varepsilon}{4 \delta t}\left\|q^{n+1}-q^{n}\right\|^{2} . \\
& -\frac{\lambda}{\delta t}\left(S-\frac{g^{\prime \prime}(\zeta)}{2},\left(\phi^{n+1}-\phi^{n}\right)^{2}\right)_{\partial \Omega} .
\end{aligned}
$$

Thus, by the assumption $S \geq \bar{L} / 2$ we get the desired energy law (3.16). 


\section{Spatial discretization}

Since the proofs of energy stability for scheme is based on weak form and integration by parts, a suitable spatial discretization method can be adopted to keep the energy dissipation properties of the semi-discretized scheme.

In this section, we consider the domain $\Omega=\left[0, L_{x}\right] \times\left[0, L_{y}\right]$, the boundary $\partial \Omega=\Gamma_{1} \cup \Gamma_{2} \cup$ $\Gamma_{3} \cup \Gamma_{4}$, and the definition of the boundary is showed in Fig. 4.1. We take the implementation

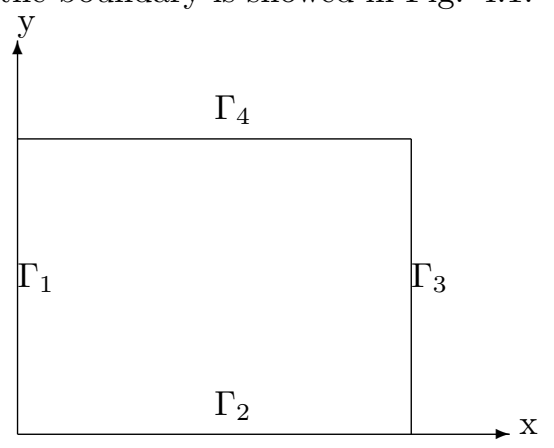

Fig. 4.1. A diagram of the boundary.

of a finite element method for the spatial discretization to test the approximation properties of our time discretization LD scheme.

\subsection{Fully discrete schemes and energy stability}

Now we consider the fully discrete scheme to solve the PDE system. Let $S_{h} \subset H^{1}(\Omega)$ be the finite-dimensional subspace, which is constructed by the piecewise linear functions.

We define $\tilde{S}_{h}^{0}=\left\{u:\left.u\right|_{\Gamma_{1} \cup \Gamma_{3}}=0, u \in S_{h}\right\}, S_{h}^{0} \subset H_{0}^{1}(\Omega)$. Let $V_{\mathbf{u}_{h}}=\tilde{S}_{h}^{0} \times S_{h}^{0}$ and $M_{h} \subset L_{0}^{2}(\Omega)$ be two finite-dimensional spaces satisfying the inf-sup condition,

$$
\inf _{q_{h} \in M_{h}} \sup _{\mathbf{u}_{h} \in V_{\mathbf{u}_{h}}} \frac{\int_{\Omega} q_{h} \nabla \cdot \mathbf{u}_{h} d x}{\left\|q_{h}\right\|\left\|\mathbf{u}_{h}\right\|_{1}} \geq C,
$$

where $C>0$ independent of mesh size $h$ and $\left\|\mathbf{u}_{h}\right\|_{1}=\left\|\nabla \mathbf{u}_{h}\right\|+\left\|\mathbf{u}_{h}\right\|$.

In the framework of the finite element spaces, the scheme (3.3)-(3.13) reads as follows.

Find $\left(\phi_{h}^{n+1}, q_{h}^{n+1}, \tilde{\mathbf{u}}_{h}^{n}, p_{h}^{n+1}, \mathbf{u}_{h}^{n+1}\right) \in S_{h} \times S_{h} \times V_{\mathbf{u}_{h}} \times M_{h} \times V_{\mathbf{u}_{h}}$, such that for all $\left(\psi_{h}, \mathbf{v}_{h}, g_{h}\right) \in$ $S_{h} \times V_{\mathbf{u}_{h}} \times M_{h}$ there holds

$$
\begin{aligned}
& \left(\frac{\phi^{n+1}-\phi^{n}}{\delta t}, \psi_{h}\right)+\left(\tilde{\mathbf{u}}_{\star h}^{n} \cdot \nabla \phi_{h}^{n}, \psi_{h}\right)+M \varepsilon\left(\nabla \phi_{h}^{n+1}, \nabla \psi_{h}\right)-M\left(\varepsilon \partial_{\mathbf{n}} \phi_{h}^{n+1}, \psi_{h}\right) \partial \Omega \\
& +M\left(\phi_{h}^{n}\left[q_{h}^{n}+\frac{2}{\varepsilon} \phi_{h}^{n}\left(\phi_{h}^{n+1}-\phi_{h}^{n}\right)\right], \psi_{h}\right)+M\left(\xi^{n}, \psi_{h}\right)=0, \\
& \left(\frac{\tilde{\mathbf{u}}_{h}^{n+1}-\mathbf{u}_{\star h}^{n}}{\delta t}, \mathbf{v}_{h}\right)+\left(B\left(\mathbf{u}_{h}^{n}, \tilde{\mathbf{u}}_{h}^{n+1}\right), \mathbf{v}_{h}\right)+\nu\left(\nabla \tilde{\mathbf{u}}_{h}^{n+1}, \nabla \mathbf{v}_{h}\right) \\
& \left.-\nu\left(\partial_{\mathbf{n}} \tilde{\mathbf{u}}_{h}^{n+1}, \mathbf{v}_{h}\right)_{\partial \Omega}+\left(\nabla p_{h}^{n}, \mathbf{v}_{h}\right)\right)=0, \\
& \left(\frac{\mathbf{u}_{h}^{n+1}-\tilde{\mathbf{u}}_{h}^{n+1}}{\delta t}, \mathbf{v}_{\mathbf{h}}\right)+\left(\nabla\left(p_{h}^{n+1}-p_{h}^{n}\right), \mathbf{v}_{h}\right)=0, \\
& \left(\nabla \cdot \mathbf{u}_{h}^{n+1}, g_{h}\right)=0 \text {, } \\
& \frac{\varepsilon}{2}\left(\frac{q_{h}^{n+1}-q_{h}^{n}}{\delta t}, \psi_{h}\right)=\left(\phi_{h}^{n} \frac{\phi_{h}^{n+1}-\phi_{h}^{n}}{\delta t}, \psi_{h}\right),
\end{aligned}
$$


where

$$
\begin{aligned}
& -\varepsilon \partial_{\mathbf{n}} \phi_{h}^{n+1}=g^{\prime}\left(\phi_{h}^{n}\right)+S\left(\phi_{h}^{n+1}-\phi_{h}^{n}\right), \\
& -\nu \partial_{\mathbf{n}} \tilde{\mathbf{u}}_{h}^{n+1}=l\left(\phi_{h}^{n}\right) \tilde{\mathbf{u}}_{s h}^{n+1} .
\end{aligned}
$$

Remark 4.1. Note that the update of $q_{h}^{n+1}$ in (4.6) is decoupled from the rest of equations.

For the convenience of establishing the stability of the fully discrete scheme (4.2)-(4.6), we introduce the discrete divergence operator $B_{h}: V_{\mathbf{u}_{h}} \rightarrow M_{h}$ such that for $\mathbf{u}_{h} \in V_{\mathbf{u}_{h}}$ and $p_{h} \in M_{h}$

$$
\left(B_{h} \mathbf{u}_{h}, p_{h}\right):=-\left(\nabla \cdot \mathbf{u}_{h}, p_{h}\right)=\left(\mathbf{u}_{h}, \nabla p_{h}\right):=\left(\mathbf{u}_{h}, B_{h}^{T} p_{h}\right),
$$

where $B_{h}^{T}: M_{h} \rightarrow V_{\mathbf{u}_{h}}$ is the transpose of $B_{h}$ (the discrete gradient operator). Hence one can write the projection step (4.4) and (4.5) in discrete form

$$
\begin{aligned}
& \mathbf{u}_{h}^{n+1}-\tilde{\mathbf{u}}_{h}^{n+1}+B_{h}^{T}\left(p_{h}^{n+1}-p_{h}^{n}\right)=0, \quad \text { in } M_{h}, \\
& B_{h} \mathbf{u}_{h}^{n+1}=0, \quad \text { in } M_{h} .
\end{aligned}
$$

Thus, the fully discrete energy of the system also satisfies the following law.

Theorem 4.1. Given that $q_{h}^{n} \in S_{h}, \phi_{h}^{n} \in S_{h}, \mathbf{u}_{h}^{n} \in V_{\mathbf{u}_{\mathbf{h}}}$, and $p_{h}^{n} \in M_{h}$, the system (4.2)-(4.6) admits a unique solution $\left(\phi_{h}^{n+1}, q_{h}^{n+1}, \mathbf{u}_{h}^{n+1}, p_{h}^{n+1}\right) \in S_{h} \times S_{h} \times V_{\mathbf{u}_{h}} \times M_{h}$ at the time $t^{n+1}$ for any parameters $h>0$ and $\delta t>0$. Moreover, by the assumption of $\mathbf{u}_{w}=0$ and $S \geq \bar{L} / 2$, the solution satisfies a discrete energy law

$$
\begin{aligned}
& E_{\text {tot }_{h}^{n+1}}^{n+1}+\frac{\delta t^{2}}{2}\left\|B_{h}^{T} p_{h}^{n+1}\right\|^{2}+\delta t\left[\nu\left\|\nabla \tilde{\mathbf{u}}_{h}^{n+1}\right\|^{2}+\frac{\lambda}{M}\left\|\dot{\phi}_{h}^{n+1}\right\|^{2}+\left\|l^{1 / 2}\left(\phi_{h}^{n}\right) \tilde{\mathbf{u}}_{s h}^{n+1}\right\|_{\partial \Omega}^{2}\right] \\
& \leq E_{\text {tot } h_{h}^{n}}+\frac{\delta t^{2}}{2}\left\|B_{h}^{T} p_{h}^{n}\right\|^{2}, \quad n=0,1,2, \cdots
\end{aligned}
$$

where $E_{t o t h}^{n}=\left\|\mathbf{u}_{h}^{n}\right\|^{2} / 2+\lambda\left(\varepsilon\left\|\nabla \phi_{h}^{n}\right\|^{2} / 2+\varepsilon\left\|q_{h}^{n}\right\|^{2} / 4+\left(g\left(\phi_{h}^{n}, 1\right)\right)_{\partial \Omega}\right)$, and $\dot{\phi}_{h}^{n+1}=\left(\phi_{h}^{n+1}-\right.$ $\left.\phi_{h}^{n}\right) / \delta t+\left(\mathbf{u}_{\star h}^{n+1} \cdot \nabla\right) \phi_{h}^{n}$.

Proof. Noting that the scheme (4.2)-(4.6) is a linear system, thus the unique solvability would follow from the energy law (4.12).

By working with (4.2), (4.3), (4.6), (4.10) and (4.11), and by the same proof of Theorem 3.1 , we can easily prove that it satisfies the energy law (4.12).

\section{Numerical simulation}

We present in this section some numerical experiments using the schemes constructed in the section 3 .

Our spatial discretization is based on the finite element method. We use the inf-sup stable Iso-P2/P1 element [44] for the velocity and pressure, and linear element for the phase function $\phi$.

Example 1: Accuracy test. We first test the convergence rates of the proposed schemes (3.3)-(3.13). In $\Omega=[0,2]^{2}$, we set the exact solution as

$$
\left\{\begin{array}{l}
\phi(t, x, y)=2+\cos (\pi x) \cos (\pi y) \sin t \\
u(t, x, y)=\pi \sin (2 \pi y) \sin ^{2}(\pi x) \sin t \\
v(t, x, y)=-\pi \sin (2 \pi x) \sin ^{2}(\pi y) \sin t \\
p(t, x, y)=\cos (\pi x) \sin (\pi y) \sin t
\end{array}\right.
$$




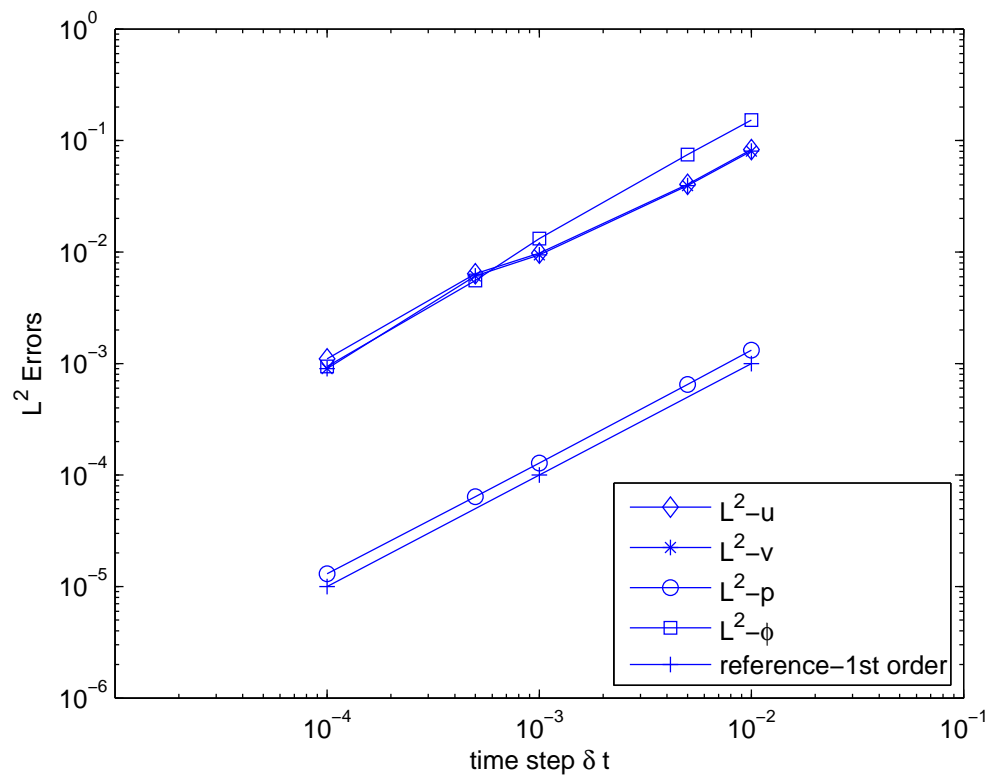

Fig. 5.1. Example 1. Temporal convergence rates of $L^{2}$ errors for the velocity $(\mathbf{u}=(u, v))$, pressure $p$, and phase field function $\phi$ in scheme (3.3)-(3.13).

We choose $\varepsilon=0.025, \nu=1, M=0.001, \lambda=10^{-7}, \gamma=1000, l(\phi)=1 /(0.19), \theta_{s}=90^{\circ}$. Some suitable force fields are imposed such that the given solutions satisfy the coupled systems. We used 10145 nodes and 19968 triangle elements in this test.

For the accuracy test, we plot the $L^{2}$ errors of the velocity, pressure and phase function between the numerical solution and the exact solution at $t=1$ with different time step size $\delta t=0.0001,0.0005,0.001,0.005$, and 0.01 in Figure 5.1. The numerical results show that scheme (3.3)-(3.13) is first-order accurate in time for all variables.

\section{Example 2: Immiscible Couette flow.}

In this case the domain is set to be $\Omega=[0,4] \times[0,0.8]$ and the solid wall velocity is set to be $V=0.7$ and $\lambda=0.1$. We choose the different value of $\theta_{s}$ and the other parameters in Example 1. We set the initial values $\mathbf{u}^{0}=0$ and

$$
\phi^{0}(x, y)= \begin{cases}1, & (x, y) \in \Gamma \\ -1, & \text { else }\end{cases}
$$

where $\Gamma$ is the initial configuration of fluid 1 , and $\Gamma=\{(x, y) \in \Omega|| x-2 \mid \leq 1\}$. We obtain 9153 nodes and 17920 triangle elements in the fixed domain produced by the PDE tool in Matlab. The Couette flow is generated by moving the top and bottom walls at a speed $V$ along $\pm x$, respectively.

In Example 2 (a), we set the contact angle $\theta_{s}=60^{\circ}$, and we see that the contact line of Fig. 5.2 is moving from $T=0.01$ to $T=3$. The contact line goes to steady state form $T=3$ to $T=5$.

In Example 2 (b), we set the angle $\theta_{s}=103^{\circ}$ on the top boundary and $\theta_{s}=77^{\circ}$ on the bottom boundary. In Fig. 5.3, we also see that the contact line is moving from $T=0.01$ to $T=3$ and reaches equilibrium state at $T=5$. Theses results is consistent to the numerical results in $[24,41]$. 
Example 3: Spreading and dewetting of a droplet.

In this example we set the domain $\Omega=[0,4] \times[0,1.2]$ and the initial configuration $\Gamma=$ $\left\{(x, y) \in \Omega \mid(x-2)^{2}+y^{2} \leq 0.64\right\}$.

We want to simulate the process of the spreading and dewetting of a droplet. The solid wall velocity is set to be $V=0$ and other parameters are set to be the same in Example 2. In this example we select the contact angle $\theta_{s}=30^{\circ}$ in the wetting case and $\theta_{s}=150^{\circ}$ in the dewetting case. We see that the contact line is spreading out from $T=0.01$ to $T=0.5$ in the right of Fig. 5.4 and goes to steady state at $T=5$ in the right of Fig. 5.5. In the dewetting case the contact line is shrinking from $T=0.01$ to $T=0.5$ in the right of Fig. 5.4 and also goes to steady state at $T=5$ in the right of Fig. 5.5. Fig. 5.6 shows that the energy of the two cases is decreasing and goes to steady state at the end. Due to the boundary energy $E_{s}(\phi)=\lambda(g(\phi), 1)_{\partial \Omega}$, the total energy in dewetting case is less than zero and that in wetting case is bigger than zero. Both cases match to the numerical results in $[24,41]$.

Example 4: A droplet in a cylinder with a hole.

We now consider the physical domain $\Omega$ to be a cylinder with a hole, and assume that all variables are axisymmetric. For the implementation of the cylindrical coordinates $(r, \theta, z)$, the Navier-Stokes equations in the system read as in $[23,32]$. Without considering the azimuthal $\theta$ direction, the system can be considered to be two-dimensional problems briefly.

In this case we select the contact angle $\theta_{s}=30^{\circ}$ for $(a)$ and $\theta_{s}=150^{\circ}$ for $(b)$, considering the effect of gravity on the system. We can use the momentum equation as follows,

$$
\mathbf{u}_{t}+(\mathbf{u} \cdot \nabla) \mathbf{u}+\nabla p=\lambda \mu \nabla \phi+\phi g_{0} e_{z},
$$

where $g_{0}$ is the gravity acceleration and $e_{z}=(0,1)$. The computation domain is $R=1, Z=2$, which has a rectangle hole. The irregular domain is discretized into 20105 nodes and 39680 triangle elements by MATLAB. The initial configuration is set to be a hole around a semicircle.

The Fig. 5.7 and Fig. 5.8 show that the droplet is dropping with time going on and the contact line is spreading. Similarly, the Fig. 5.9 and Fig. 5.10 show that the droplet is dropping and the contact line is shrinking.

\section{Conclusions and remarks}

In this paper we studied the Navier-Stokes coupled with Allen-Cahn phase field model for two phase immiscible complex fluids. With the implementation of the general Navier boundary condition and the dynamic condition, we derived the energy dissipation law for the model. We then constructed a linear decoupled energy stable scheme by the introduction of the auxiliary function.

We have not only considered time discretizations here, but also take the implementation on the spatial dicretizations by the finite element method. Moreover, we prove that the fully discrete scheme is also energy stable. At last we presented ample numerical results to validate the numerical scheme and illustrate the contact lines' movements, wetting and dewetting phenomena.

\section{Acknowledgments}

R. Chen is partially supported by the China Postdoctoral Science Foundation grant No. 2016M591122. X. Yang is partially supported by NSF DMS-1200487, NSF DMS-1418898, 
AFOSR FA9550-12-1-0178. H. Zhang is partially supported by NSFC/RGC Joint Research Scheme No. 11261160486, NSFC grant No. 11471046, 11571045 and the Ministry of Education Program for New Century Excellent Talents Project NCET-12-0053.

\section{References}

[1] K. Bao, Y. Shi, S, Sun and X.P. Wang, A finite element method for the numerical solution of the coupled Cahn-Hilliard and Navier-Sotkes system for moving contact line problems, J. Comput. Phys., 231 (2012), 8083-8089.

[2] F. Boyer and C. Lapuerta, Study of a three component Cahn-Hilliard flow model, ESAIM: Math. Model. Num. Ana., 40 (2006), 653-687.

[3] F. Boyer and S. Minjeaud, Numerical schemes for a three component Cahn-Hilliard model, ESAIM Math. Model. Numer. Anal., 45 (2011), 697-738.

[4] R. Chen, G. Ji, X. Yang, and H. Zhang, Decoupled energy stable schemes for phase-field vesicle membrane model, J. Comput. Phys., 302 (2015), 509-523.

[5] Y. Gong, J. Zhao, X. Yang and Q. Wang, A novel linear second order unconditionally energy stable scheme for a hydrodynamic q-tensor model of liquid crystals. in press, DOI: 10.1016/j.cma.2017.01.031, Comput. Meth. Appl. Mech. Engrg., 2017.

[6] J. L. Guermond, P. Minev, and J. Shen, An overview of projection methods for incompressible flows, Comput. Meth. Appl. Mech. Engrg., 195 (2006), 6011-6045.

[7] J. L. Guermond, J. Shen, and X. Yang, Error analysis of fully discrete velocity-correction methods for incompressible flows, Math. Comp., 77 (2008), 1387-1405.

[8] N.G. Hadjiconstantinou, Hybrid atomistic-continuum formulations and the moving contact-line problem, J. Comput. Phys., 154 (1999), 245-265.

[9] D. Han, A. Brylev, X. Yang, and Z. Tan, Numerical analysis of second order, fully discrete energy stable schemes for phase field models of two phase incompressible flows, J. Sci. Comput., 70 (2017), 965-989.

[10] Q. He, R. Glowinski and X.P. Wang, A least-squares/finite dlement method for numerical solution of the Navier-Stokes-Cahn-Hilliard system modeling the motion of the contact line, J. Comput. Phys., 230 (2011), 4991-5009.

[11] J. Hua, P. Lin, C. Liu, and Q. Wang, Energy law preserving c0 finite element schemes for phase field models in two-phase flow computations, J. Comput. Phys., 230 (2011), 7155-7131. Mech. Engrg., 2017.

[12] M. Kapustina, D.Tsygakov, J. Zhao, J.Wessler, X. Yang, A. Chen, N. Roach, T. C. Elston, Q.Wang, K. Jacobson, and M. G. Forest, Modeling the excess cell surface stored in a complex morphology of bleb-like protrusions, PLOS Computational Biology, 12 (2016), e1004841.

[13] J. Kim, Phase field computations for ternary fluid flows, Comput. Meth. Appl. Mech. Engrg., 196 (2007), 4779-4788.

[14] J. Kim and J. Lowengrub, Phase field modeling and simulation of three-phase flows, Interfaces and Free Boundaries, 7 (2005), 435-466.

[15] J. Koplik, J.R. Banavar and J.F. Willemsen, Molecular dynamics of poiseuille flow and moving contact lines, Phys. Rev. Lett., 60 (1988), 1282-1285.

[16] J. Koplik, J.R. Banavar and J.F. Willemsen, Molecular dynamics of fluid flow at solid surfaces, Phys. Fluids A. Fluid Dyn., 1 (1989), 781-794.

[17] Z. Li, M.C. Lai, G. He and H. Zhao, An augmented method for free boundary problems with moving contact lines, Comput. Fluids, 39 (2010), 1033-1040.

[18] J. Li and Q. Wang, Mass conservation and energy dissipation issue in a class of phase field models for multiphase fluids, J. Appl. Mech., 81 (2013), 021004.

[19] T. S. Little, V. Mironov, A. Nagy-Mehesz, R. Markwald, Y. Sugi, S. M. Lessner, M. A. Sutton, X. Liu, Q. Wang, X. Yang, J. O. Blanchette, and M. Skiles, Engineering a 3d, biological construct: 
representative research in the south carolina project for organ biofabrication, Biofabrication, $\mathbf{3}$ (2011), 030202.

[20] C. Liu and J. Shen, A phase field model for the mixture of two incompressible fluids and its approximation by a Fourier-spectral method, Physica D, 179 (2003), 211-228.

[21] C. Liu, J. Shen and X. Yang, Dynamics of defect motion in nematic liquid crystal flow: modeling and numerical simulation, Commun. Comput. Phys., 2 (2007), 1184-1198.

[22] C. Liu, J. Shen and X. Yang, Decouppled Energy stable schemes for a phase-field model of twophase incompressible flows with variable density, SIAM J. Sci. Comput., 62 (2015), 601-622.

[23] J.M. Lopez, F. Maques and J. Shen, An efficent spectral-projection method for the Navier-Stokes equations in cylidrical geometries, J. Comput. Phys., 139 (1998), 308-326.

[24] L. Ma, R. Chen, X. Yang and H. Zhang, Numerical approxiamations for Allen-Cahn type phase field model of two-phase incompressible fluids with moving contact line, Commun. Comput. Phys., 21 (2017), 867-889.

[25] C. Miehe, M. Hofacker, and F. Welschinger, A phase field model for rate-independent crack propagation: Robust algorithmic implementation based on operator splits, Comput. Meth. Appl. Mech. Engrg., 199 (2010), 2765-2778.

[26] S. Minjeaud, An unconditionaly stable uncoupled shceme for a triphasic Cahn-Hilliard/NavierStokes model, Commun. Comput. Phys., 29 (2013), 584-618.

[27] N. Moelans, B. Blanpain, and P. Wollants, A phase field model for grain growth and thermal grooving in thin films with orientation dependent surface energy, Solid State Phenomena, 129 (2007),89-94.

[28] T. Qian, X. Wang and P. Shen, Molecular scale contact line hydrodyanmics of immiscible flows, Phys. Rev. E, 68 (2003), 016306.

[29] T. Qian, X. Wang and P. Shen, A variational approach to moving contact line hydrodynamics, J. Fluid Mech., 564 (2006), 333-360.

[30] W. Ren and W. E, Heterogeneous multiscale method for the modeling of complex fluids and micro-fluids, J. Comput. Phys., 204 (2005), 1-26.

[31] M. Renardy, Y. Renardy and J. Li, Numerical simulation of moving contact line problems using a volume of fluid method, J. Comput. Phys., 171 (2001), 243-263.

[32] J. Shen, Efficient spectral-galerkin method III:polar and cylindrical geometries, SIAM J. Sci. Comput., 18 (1997), 1583-1604.

[33] J. Shen, C. Wang, X. Wang and S.M. Wise, Second-order converx splitting schemes for gradient flows with Ehrlich-Schewoebel type energy: application to thin film epitaxy, SIAM J. Numer. Anal., 50 (2012), 105-125.

[34] J. Shen and X. Yang, Error estimates for finite element approximations of consistent splitting schemes for incompressible flows, Disc. Conti. Dyn. Sys.-B, 8 (2007), 663-676.

[35] J. Shen and X. Yang, An efficient moving mesh spectral method for the phase-field model of two-phase flows, J. Comput. Phys., 228 (2009), 2978-2992.

[36] J. Shen and X. Yang, Energy stable schemes for CahnCHilliard phase-field model of two-phase incompressible flows, Chin. Ann. Math. Ser. B, 31 (2010), 743-758.

[37] J. Shen and X. Yang, Numerical approximations of AllenCCahn and CahnCHilliard equations, Disc. Cont. Dyn. Sys. A, 28 (2010), 1669-1691.

[38] J. Shen and X. Yang, Decoupled energy stable schemes for phase filed models of two phase complex fluids, SIAM J. Sci. Comput., 36 (2014), B122-B145.

[39] J. Shen and X. Yang, Decoupled, energy stable schemes for phase-field models of two-phase incompressible flows, SIAM J. Num. Anal., 53 (2015), 279-296.

[40] J. Shen, X. Yang, and Q. Wang, On mass conservation in phase field models for binary fluids, Comm. Compt. Phys, 13 (2012), 1045-1065.

[41] J. Shen, X. Yang, and H. Yu, Efficient energy stable numerical schemes for a phase field moving contact line model, J. Comput. Phys., 284 (2015), 617-630. 
[42] R. Spatschek, E. Brener, and A. Karma, A phase field model for rate-independent crack propagation: Robust algorithmic implementation based on operator splits, Philos. Mag., 91 (2010), 75-95.

[43] R. Spatschek, M. Hartmann, E. Brener, H. Muller-Krumbhaar, and K. Kassner, Phase field modeling of fast crack propagation, Phys. Rev. Lett., 96 (2006), 015502.

[44] M. Tabata and D. Tagamai, Error estimates for finite element approximations of drag and lift in nonstantionary Navier-stokes flows, Jpn. J. Indust. Appl. Math., 17 (2000), 371-389.

[45] R. Témam, Sur l'approximation de la solution des équations de Navier-Stokes pae lameéthode des pas fractionnaries II, Arch. Rational Mech. Anal., 33 (1969), 377-385.

[46] L. Vanherpe, N. Moelans, B. Blanpain, and S. Vandewalle, Bounding box framework for efficient phase field simulation of grain growth in anisotropic systems, Comput. Materials Sci., 50 (2011), 2221-2231.

[47] C. Wang and S. M. Wise, An energy stable and convergent finite-difference scheme for the modified phase field crystal equation, SIAM J. Numer. Anal., 49 (2011), 945-969.

[48] Q. Wang, X. Yang, D. Adalsteinsson, T. C. Elston, K. Jacobson, M. Kapustina, and M. G. Forest, Computational and modeling strategies for cell motility. Book chapter in Computational Moleculear cell modeling, Part of the series Biological and Medical Physics, Biomedical Engineering, Edited by Nikolay Dokholyan, Spring, New York, pages 257-296, (2012).

[49] X. Yang, Error analysis of stabilized semi-implicit method of Allen-Cahn equation, Disc. Conti. Dyn. Sys.-B, 11 (2009), 1057-1070.

[50] X. Yang, Linear, first and second order and unconditionally energy stable numerical schemes for the phase field model of homopolymer blends, J. Comput. Phys., 327 (2016), 294-316.

[51] X. Yang, J. J. Feng, C. Liu, and J. Shen, Numerical simulations of jet pinching-off and drop formation using an energetic variational phase-field method, J. Comput. Phys., 218 (2006), 417428.

[52] X. Yang, M. G. Forest, H. Li, C. Liu, J. Shen, Q. Wang, and F. Chen, Modeling and simulations of drop pinch-off from liquid crystal filaments and the leaky liquid crystal faucet immersed in viscous fluids, J. Comput. Phys., 236 (2013), 1-14.

[53] X. Yang and D. Han, Linearly first- and second-order, unconditionally energy stable schemes for the phase field crystal equation, J. Comput. Phys., 330 (2017), 1116-1134.

[54] X. Yang and L. Ju, Efficient linear schemes with unconditionally energy stability for the phase field elastic bending energy model, Comput. Meth. Appl. Mech. Engrg., 315 (2017), 691-712.

[55] X. Yang and J. Lu, Linear and unconditionally energy stable schemes for the binary fluid-surfactant phase field model, Comput. Meth. Appl. Mech. Engrg., 318 (2017), 1005-1029.

[56] X. Yang, Q. Wang, and V. Mironov, Modeling fusion of cellular aggregates in biofabrication using phase field theories, J. Theoret. Biol., 303 (2011), 110-118.

[57] X. Yang, J. Zhao, and Q. Wang, Numerical approximations for the molecular beam epitaxial growth model based on the invariant energy quadratization method, J. Comput. Phys., 333 (2017), 104-127.

[58] X. Yang, J. Zhao, Q. Wang, and J. Shen, Numerical approximations for a three components CahnHilliard phase-field model based on the invariant energy quadratization method, arXiv:1701.07469, (2017)

[59] H. Yu and X. Yang, Decoupled energy stable schemes for phase field model with contact lines and variable densities, J. Comput. Phys., 334 (2017), 665-686.

[60] J. Zhao, H. Li, Q. Wang, and X. Yang, A linearly decoupled energy stable scheme for phase-field models of three-phase incompressible flows, J. Sci. Comput., 70 (2017), 1367-1389.

[61] J. Zhao, Q. Wang, and X. Yang, Numerical approximations to a new phase field model for immiscible mixtures of nematic liquid crystals and viscous fluids, Comput. Meth. Appl. Mech. Engrg., 310 (2016), 77-97.

[62] J. Zhao, Q. Wang, and X. Yang, Numerical approximations for a phase field dendritic crystal 
growth model based on the invariant energy quadratization approach, Doi: 10.1002/nme.5372, in press, Inter. J. Num. Meth. Engr., (2017).

[63] J. Zhao, X. Yang, J. Li, and Q. Wang, Energy stable numerical schemes for a hydrodynamic model of nematic liquid crystals, SIAM. J. Sci. Comput., 38 (2016), A3264-A3290.

[64] J. Zhao, X. Yang, J. Shen, and Q. Wang, A decoupled energy stable scheme for a hydrodynamic phase-field model of mixtures of nematic liquid crystals and viscous fluids, J. Comput. Phys., 305 (2016), 539-556. 


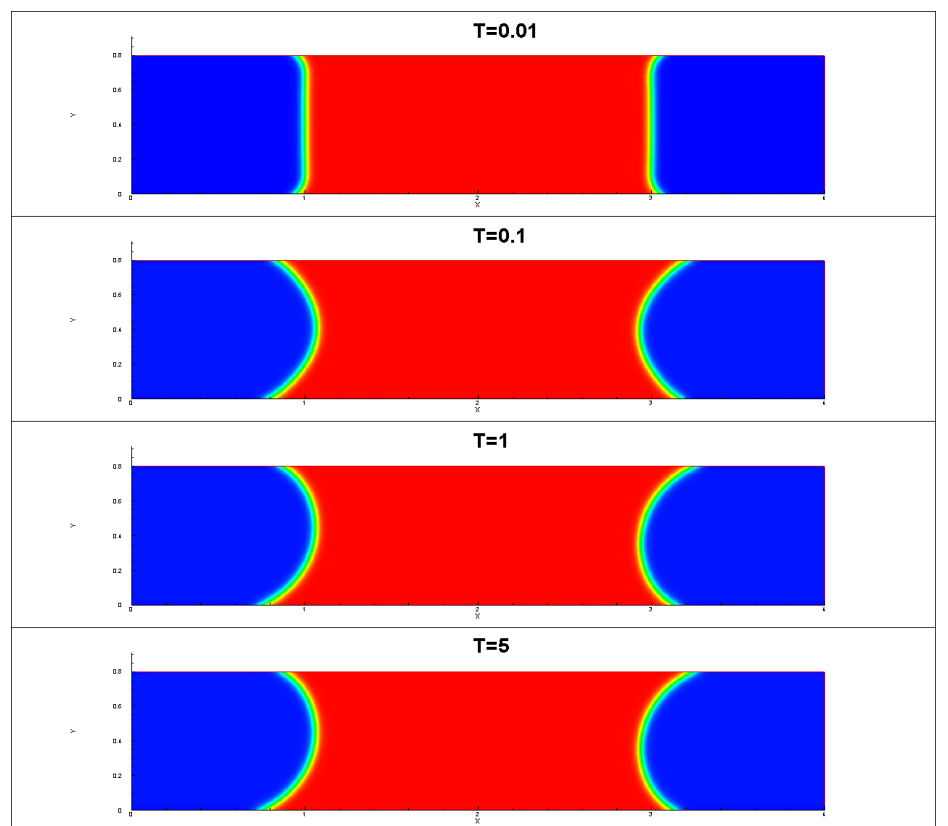

Fig. 5.2. Example 2 (a): The motion of the two fluids at the time of $T=0.01,0.1,1,5$ with contact angle $\theta_{s}=60^{\circ}$.

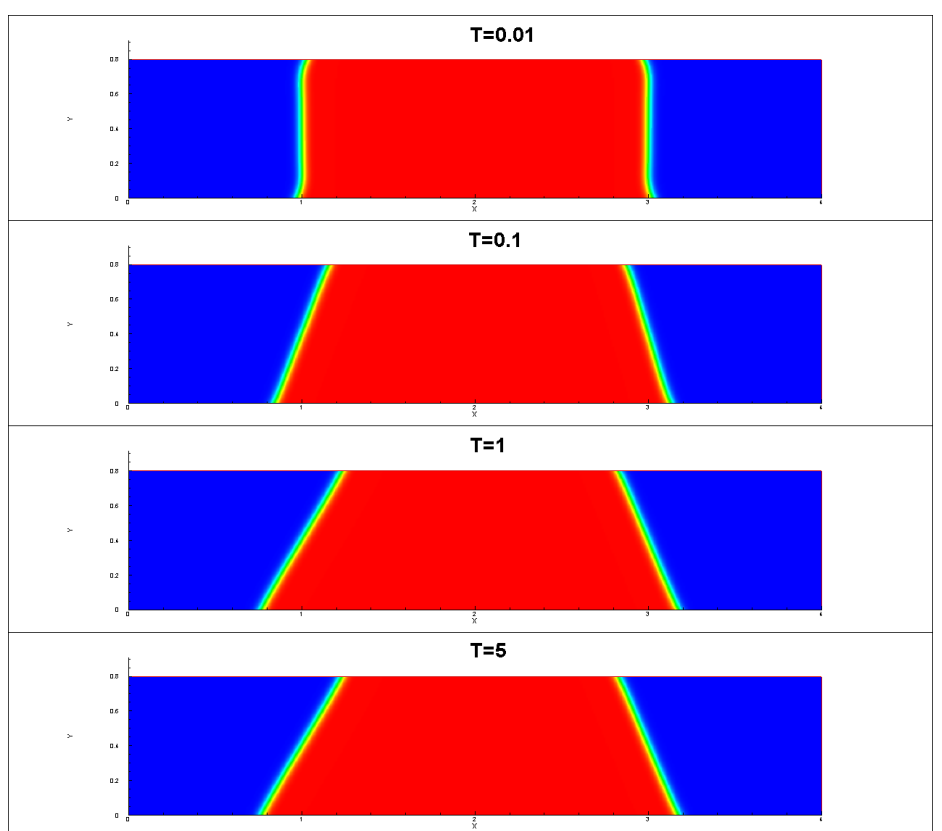

Fig. 5.3. Example 2 (b): The motion of the two fluids at the time of $T=0.01,0.1,1,5$ with contact angle $\theta_{s}=103^{\circ}$ on the top and $\theta_{s}=77^{\circ}$ on the bottom. 


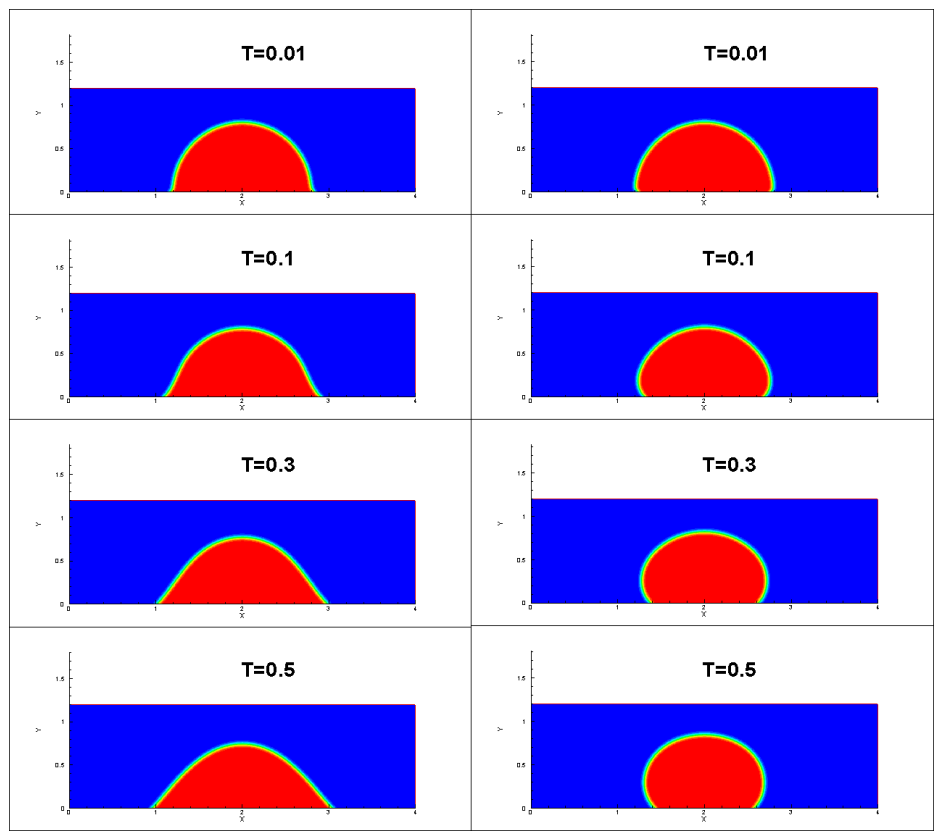

Fig. 5.4. Example 3: The motion of the two fluids at the time of $T=0.01,0.1,0.3,0.5$ with the different contact angle. Left: $\theta_{s}=30^{\circ}$, right: $\theta_{s}=150^{\circ}$.

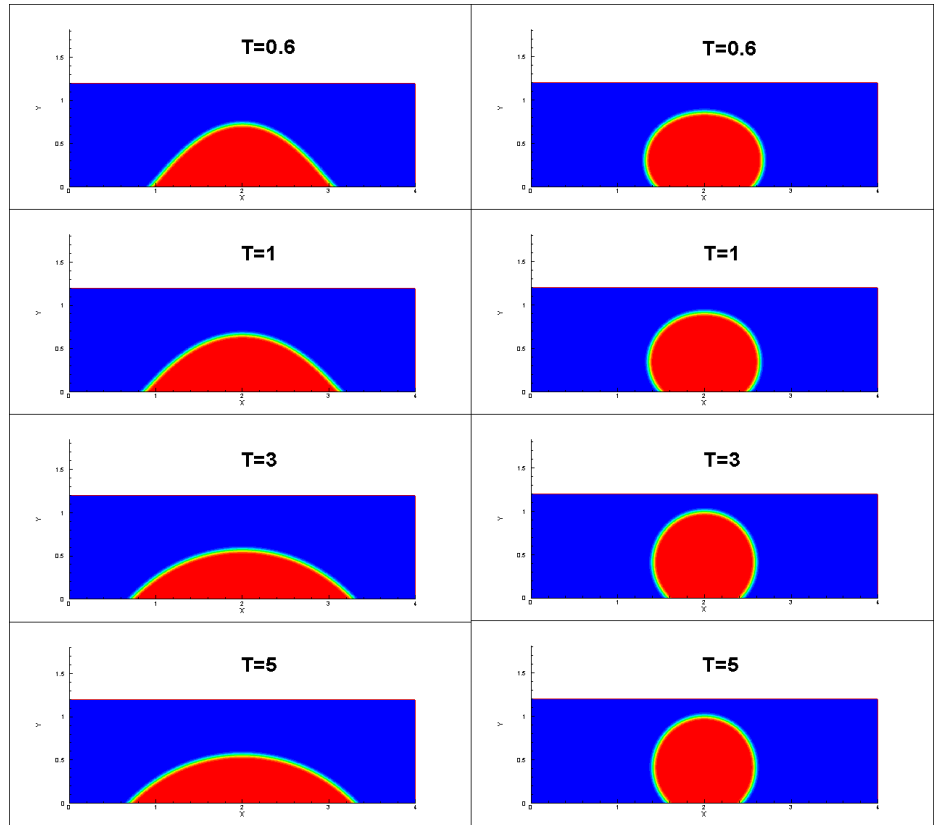

Fig. 5.5. Example 3: The motion of the two fluids at the time of $T=0.6,1,3,5$ with the different contact angle. Left: $\theta_{s}=30^{\circ}$, right: $\theta_{s}=150^{\circ}$. 


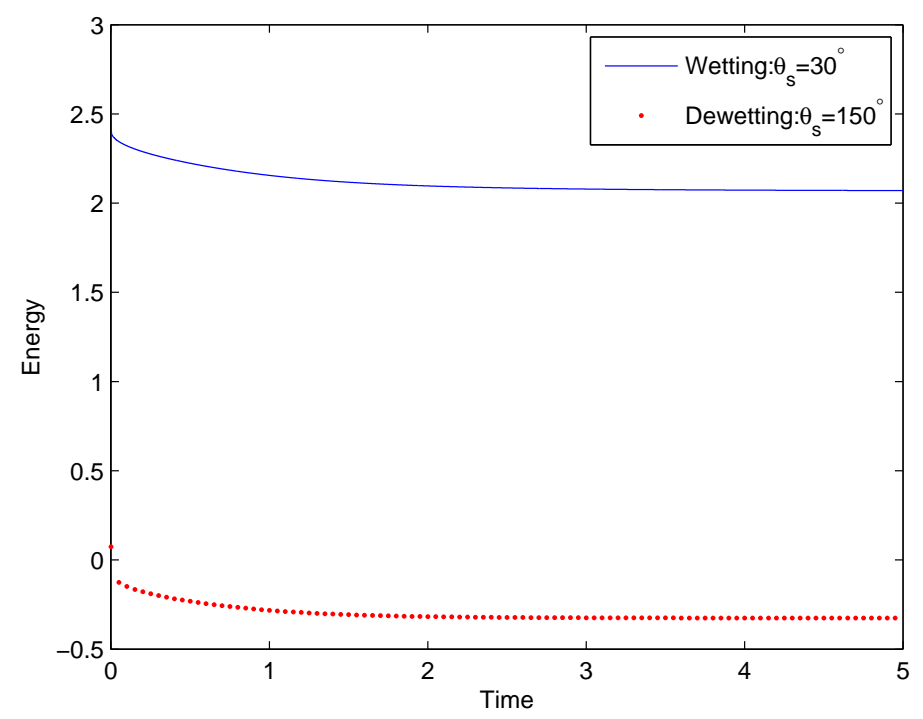

Fig. 5.6. Example 3: Time evolution of the free energy functional for wetting case $\theta_{s}=30^{\circ}$ and dewetting case $\theta_{s}=150^{\circ}$. The energy curves of the two cases are decreasing and reach the steady state at the end.

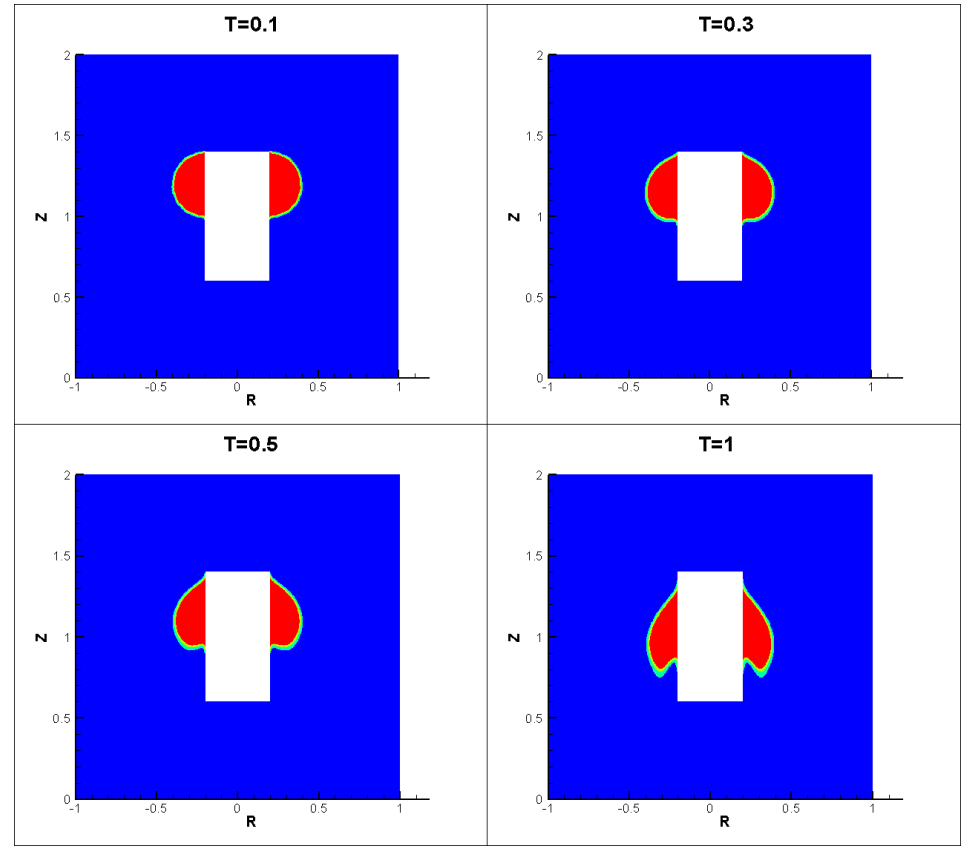

Fig. 5.7. Example 4(a): The motion of the two fluids at the time of $T=0.1,0.3,0.5,1$ with the contact angle $\theta_{s}=30^{\circ}$. 


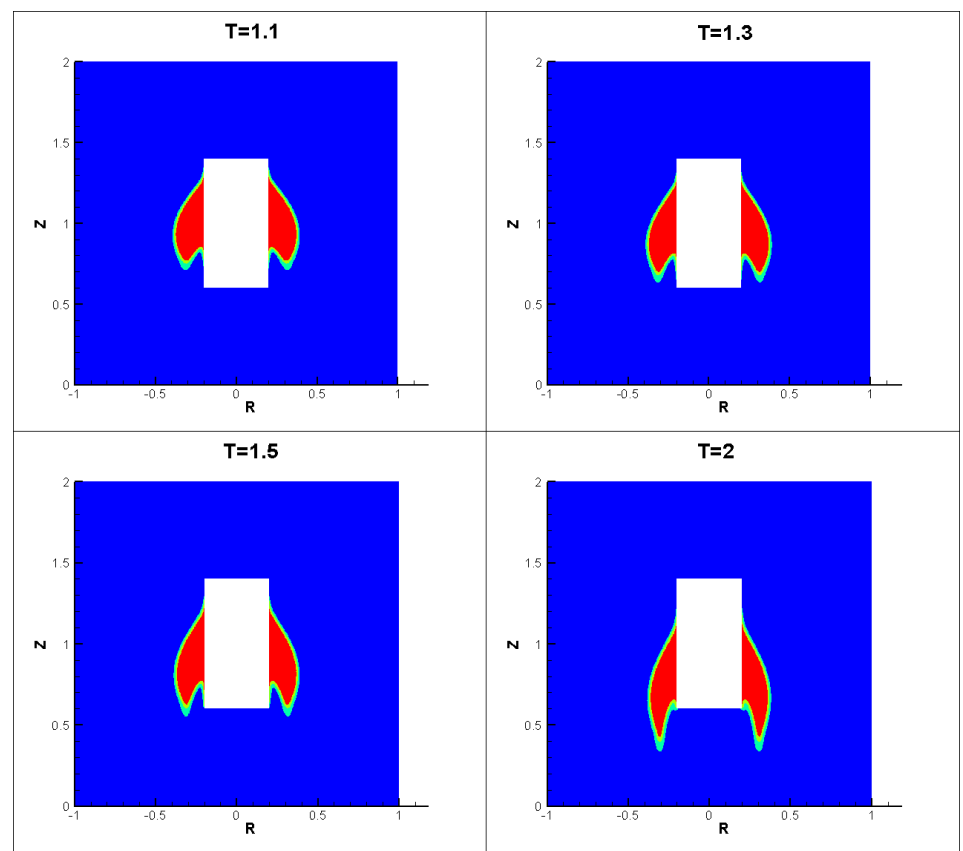

Fig. 5.8. Example 4(a): The motion of the two fluids at the time of $T=1.1,1.3,1.5,2$ with the contact angle $\theta_{s}=30^{\circ}$.

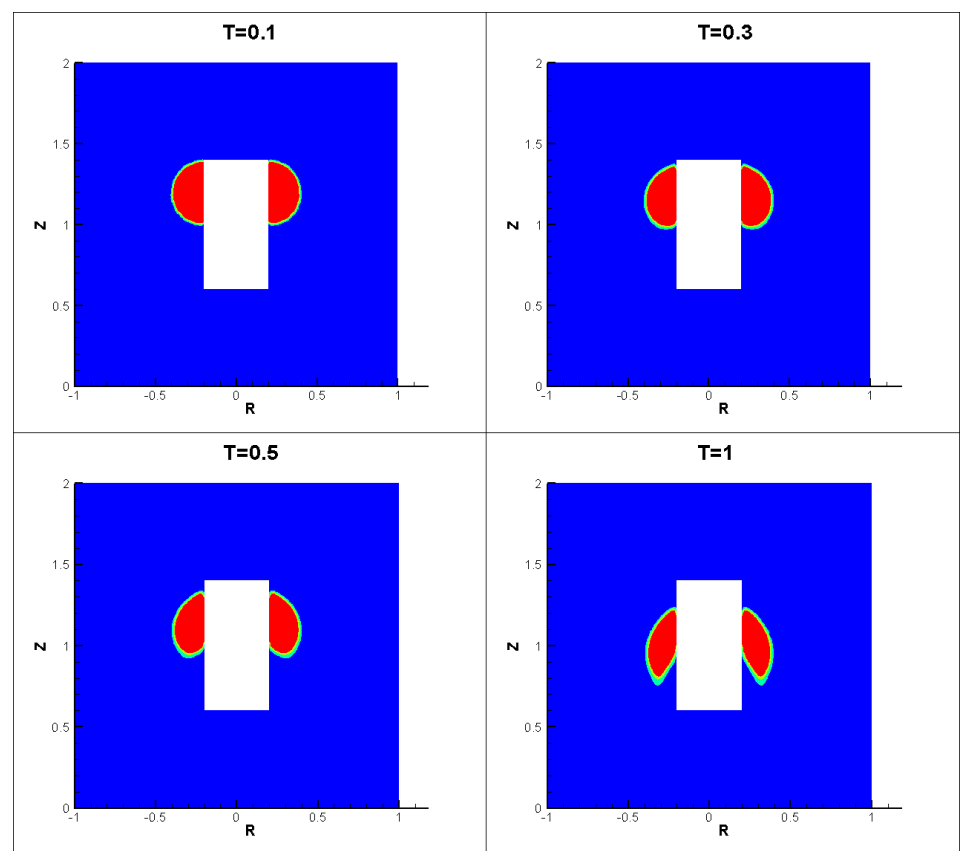

Fig. 5.9. Example 4(b): The motion of the two fluids at the time of $T=0.1,0.3,0.5,1$ with the contact angle $\theta_{s}=150^{\circ}$. 


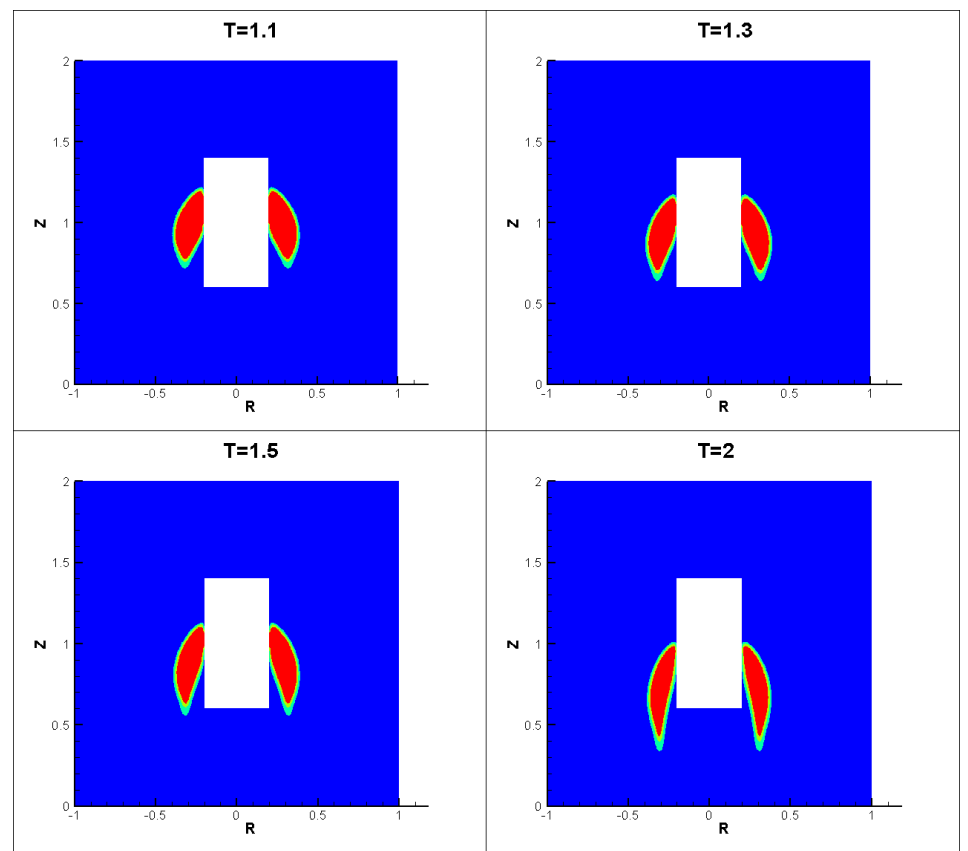

Fig. 5.10. Example 4(b): The motion of the two fluids at the time of $T=1.1,1.3,1.5 .2$ with the contact angle $\theta_{s}=150^{\circ}$. 